\title{
A Numerical Well-Balanced Scheme for One-Dimensional Heat Transfer in Longitudinal Triangular Fins
}

\author{
I. Rusagara and C. Harley \\ Centre for Differential Equations, Continuum Mechanics and Applications, School of Computational and Applied Mathematics, \\ University of the Witwatersrand, Johannesburg, Private Bag 3, 2050, South Africa
}

Correspondence should be addressed to C. Harley; charis.harley@wits.ac.za

Received 6 March 2013; Accepted 10 April 2013

Academic Editor: Fazal M. Mahomed

Copyright (C) 2013 I. Rusagara and C. Harley. This is an open access article distributed under the Creative Commons Attribution License, which permits unrestricted use, distribution, and reproduction in any medium, provided the original work is properly cited.

\begin{abstract}
The temperature profile for fins with temperature-dependent thermal conductivity and heat transfer coefficients will be considered. Assuming such forms for these coefficients leads to a highly nonlinear partial differential equation (PDE) which cannot easily be solved analytically. We establish a numerical balance rule which can assist in getting a well-balanced numerical scheme. When coupled with the zero-flux condition, this scheme can be used to solve this nonlinear partial differential equation (PDE) modelling the temperature distribution in a one-dimensional longitudinal triangular fin without requiring any additional assumptions or simplifications of the fin profile.
\end{abstract}

\section{Introduction}

There is a wide range of applications for extended surfaces, mostly called fins, in heat transfer which is more formally described as the temperature propagation or flow of the heat. Obvious examples may be found in several applications of mechanical engineering and in many home appliances [1]. In support of their use, Sparrow and Vemuri [2] have shown that with finned surfaces the heat transfer increases six times in comparison to unfinned surfaces. It has been found by many researchers that the fin orientation, height, length, and spacing in arrays play major roles in the manner and efficiency of heat transfer [3-8]. Given that these parameters play a fundamental role in the structure of the problem, and even though these interlinked factors increase the complexity of the problem, they should not be removed for the sake of simplicity. The consequence of this, however, is that we end up considering a nonlinear PDE, the solution of which is not always possible analytically. In fact, the use of analytical methods has often led to the consideration of a simplified model, especially for complex geometries, whereas this is not necessary when using certain numerical methods. However, as suggested by Wang et al. [9], when using numerical schemes as the solution method, it is fundamental to recognize that a proper numerical treatment of the source term may eliminate possible spurious steady-state numerical solutions. In this paper we will follow this line of thought and thus specifically focus on an appropriate treatment of the source term of the problem under consideration. As such, the well-balancing approach will be considered and implemented as per the work of LeVeque [10]. More precisely, this approach is applied to triangular fins which have been characterized by singularities in the literature $[11,12]$.

When solving the problem of heat transfer in a triangular fin, it is essential to remember that triangular fin profiles have been classified by Kraus et al. [11] among singular profiles because it is analytically impossible to characterize them by any linear transformation. Kraus proposes that one assume the triangular profiles to be trapezoidal in nature so as to render the problem solvable. In this manner the original problem is oversimplified so as to guarantee solutions; however, this methodology may lead to inaccurate results given that fins with trapezoidal profiles have already been classified as such and solved accordingly. Therefore, the objective of this paper is to provide a numerical approach that effectively deals with the proper form of the triangular fin and solve, via a wellbalancing numerical scheme, the problem of heat transfer in such a fin. 
In this work we use the finite volume method and illustrate how it reduces the order of differentiation by one. In this manner, by using volume averaging and the Taylor series expansion, we are able to obtain a numerical balance law. As described by Gosse and Wang $[9,13,14]$, we establish a balance law from information obtained through a consideration of the steady-state equation, which in turn is incorporated into the transient heat transfer equation. This approach is referred to as the well-balancing technique and maintains steady-state solutions. It is through this approach that we will obtain solutions to the unsteady heat transfer problem for a triangular fin.

Contrary to the suggestion made by Kraus et al. [11] that the profile of the triangular fin should be altered in order to solve the problem under consideration, we maintain the original profile, and when implementing the well-balancing approach we incorporate the zero-flux condition. In much research, the triangular fin has been considered in an inappropriate manner due to a misunderstanding of the unusual physics of the problem, especially when pertaining to the tip of the fin. Through a consideration of unsuitable boundary conditions, the numerical solution of the problem has led to inaccurate and unusual results-see [12] for such discussions. Through an incorporation of the zero-flux boundary condition, however, we eliminate any additional assumptions which would usually be required in order to solve the PDE. Rather, we establish a numerical well-balanced scheme via the incorporation of the zero-flux condition, and we validate the results obtained through the use of benchmark results $[12,15,16]$. This method of solution is novel and to the authors' best knowledge has not been used in the literature to solve the problem of singular fins. Furthermore, the approach used can easily be applied to other singular profiles such as the concave parabolic and convex parabolic profiles.

\section{Model}

We consider a longitudinal one-dimensional fin with a profile area $A_{p}$. The perimeter of the fin is denoted by $P$ and the length of fin by $L$. The fin is attached to a fixed base surface of temperature $T_{b}$ and extends into a fluid of temperature $T_{a}$. The fin profile is given by the function $F(X)$, and the fin thickness at the base is $\delta_{b}$. The energy balance for a longitudinal fin is given by [11]

$$
\begin{aligned}
\rho c_{v} \frac{\partial T}{\partial t}= & A_{p} \frac{\partial}{\partial x}\left(F(X) K(T) \frac{\partial T}{\partial x}\right) \\
& -P H(T)\left(T-T_{a}\right), \quad 0<X<L,
\end{aligned}
$$

where $K$ and $H$ are the nonuniform thermal conductivity and heat transfer coefficient depending on the temperature (see, e.g., [17-20]). We define $\rho$ as the density and $c_{v}$ as the volumetric heat capacity. The fin length is measured from the tip to the base as shown in Figure 1 (see also $[11,18,19])$.

An insulated fin at one end with the base temperature at the other implies boundary conditions which are given by [11]

$$
T(t, L)=T_{b},\left.\quad \frac{\partial T}{\partial X}\right|_{X=0}=0,
$$

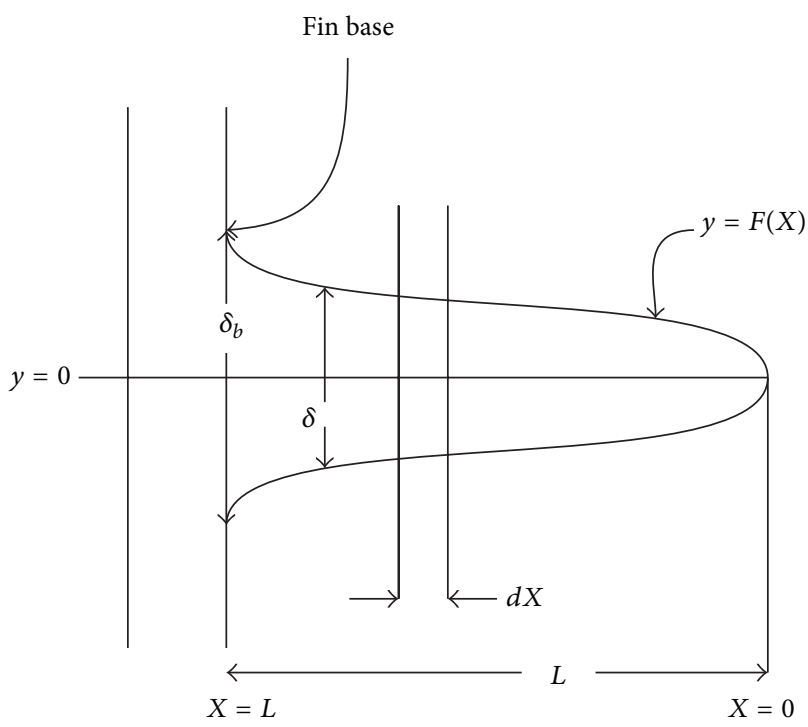

FIGURE 1: Schematic representation of a longitudinal fin with arbitrary profile $F(X)$.

and initially the fin is kept at the temperature of the fluid (the ambient temperature):

$$
T(0, X)=T_{a} .
$$

Introducing the dimensionless variables

$$
\begin{gathered}
x=\frac{X}{L}, \quad \tau=\frac{k_{a} t}{\rho c_{v} L^{2}}, \quad \theta=\frac{T-T_{a}}{T_{b}-T_{a}}, \\
h=\frac{H}{h_{b}}, \quad k=\frac{K}{k_{a}}, \\
\mathscr{M}^{2}=\frac{2 P h_{b} L^{2}}{k_{a} \delta_{b} A_{p}}, \quad f(x)=\frac{2}{\delta_{b}} F(X),
\end{gathered}
$$

then (1) reduces to the relevant dimensionless energy equation given by

$$
\frac{\partial \theta}{\partial \tau}=\frac{\partial}{\partial x}\left[f(x) k(\theta) \frac{\partial \theta}{\partial x}\right]-\mathscr{M}^{2} \theta h(\theta), \quad 0<x<1
$$

The above equation represents the nonlinear heat transfer equation when the thermal conductivity and heat transfer coefficients depend on temperature. The heat transfer coefficient is given by the power law used in most of the industrial applications $[12,21]$ as

$$
H(T)=h_{b}\left(\frac{T-T_{a}}{T_{b}-T_{a}}\right)^{n}
$$

The exponent $n$ varies between -6.6 and 5; however, it tends to lie between -3 and 3 in most practical applications [12]. Furthermore, the thermal conductivity of the fin is assumed 
to vary linearly with the temperature [12] as is the case for many engineering applications. As such we find that

$$
K(T)=k_{a}\left[1+\beta\left(T-T_{a}\right)\right]
$$

which in dimensionless variables gives $k(\theta)=1+B \theta$ where $B=\beta\left(T_{b}-T_{a}\right)$ is nonzero with $\beta$ as the thermal conductivity gradient. Hence, the dimensionless heat transfer equation for a longitudinal one-dimensional fin is given by $[12,16,22]$

$$
\frac{\partial \theta}{\partial \tau}=\frac{\partial}{\partial x}\left(f(x)(1+B \theta) \frac{\partial \theta}{\partial x}\right)-\mathscr{M}^{2} \theta^{n+1}, \quad 0<x<1, \tau \geq 0
$$

where boundary conditions are as follows:

$$
\begin{aligned}
& \left.\frac{\partial \theta}{\partial x}\right|_{x=0}=0 \text { at the fin tip, } \\
& \theta(\tau, 1)=1, \text { at the base }
\end{aligned}
$$

with initial condition

$$
\theta(0, x)=0
$$

\section{Numerical Approach}

3.1. The Finite Volume Method and Numerical Balance Law. At first we intend to briefly introduce the manner in which we will employ the finite volume method (FVM) and its advantages within the context of heat transfer problems. In this scenario, due to its integral approach, the FVM reduces the order of the spatial derivative by one. This motivates its use for the heat transfer equation, under consideration given the presence of a second derivative in its conduction term. If we were to consider the method for a more general structure of the heat transfer equation, we would consider the partial differential equation of the form

$$
\frac{\partial T}{\partial t}-\frac{A_{p}}{\rho c_{v}} \frac{\partial}{\partial x}\left(G(T, X) \frac{\partial T}{\partial x}\right)=-\frac{1}{\rho c_{v}} Q(H(T)),
$$

where $G(T, X)=F(X) K(T)$ is a function of thermal conductivity $K$, involved in the convective term, and $Q(H(T))$ a function of the heat transfer coefficient $H$, which represents the source term from radiation. Furthermore, we define $\rho$ as the density, $c_{v}$ as the heat capacity, and $T$ as the temperature.

In order to reduce the order of the spatial derivatives by one, we integrate (11) over the grid cell $\left[x_{i-1 / 2}, x_{i+1 / 2}\right]$ to obtain

$$
\begin{gathered}
\int_{x_{i-1 / 2}}^{x_{i+1 / 2}} \frac{\partial T}{\partial t} d x-\frac{A_{p}}{\rho c} \int_{x_{i-1 / 2}}^{x_{i+1 / 2}} \frac{\partial}{\partial x}\left(G(T, X) \frac{\partial T}{\partial x}\right) d x \\
=-\frac{1}{\rho c} \int_{x_{i-1 / 2}}^{x_{i+1 / 2}} Q(H(T)) d x .
\end{gathered}
$$

By cell averaging we find that

$$
\Delta x_{i} \frac{d \widetilde{T}_{i}(t)}{d t}-\left.\frac{A_{p}}{\rho c}\left(G(T, X) \frac{\partial T}{\partial x}\right)\right|_{x_{i-1 / 2}} ^{x_{i+1 / 2}}=-\Delta x_{i} \frac{1}{\rho c} \widetilde{Q}_{i},
$$

where

$$
\widetilde{f}_{i}=\frac{1}{\Delta x_{i}} \int_{x_{i-1 / 2}}^{x_{i+1 / 2}} f d x
$$

is the cell-averaged quantity of $f$ over the grid cell $\left[x_{i-1 / 2}\right.$, $\left.x_{i+1 / 2}\right]$. It is obvious that the order of the partial differential equation under consideration has been reduced by one, and this increases the accuracy of the results we are to obtain.

In the next section, we will employ the numerical approach described above for (8) and in doing so develop a numerical balance law as given by (14). In this manner, we obtain a well-balanced scheme which preserves specific nontrivial steady-state solutions and may help to minimize some of the oscillations which occur around steady states [23]. Thus for the more general heat transfer equation (11) a well-balanced scheme can provide a solution that must satisfy

$$
A_{p} \frac{\partial}{\partial x}\left(G(T, X) \frac{\partial T}{\partial x}\right)=Q(H(T))
$$

for steady states. An easily understandable and effective procedure has been established by Wang et al. [9] which will be implemented in this work for the one-dimensional heat transfer problem given by (8). It should also be kept in mind that this methodology may easily be extended to higher dimensions.

3.2. Numerical Well-Balanced Scheme. In considering (8) we find that the one-dimensional steady heat equation for regular fins is expressed by

$$
\begin{gathered}
\frac{d}{d x}\left(f(x)(1+B \theta) \frac{d \theta}{d x}\right)=\mathscr{M}^{2} \theta^{n+1}, \quad 0<x<1, \\
\left.\frac{d \theta}{d x}\right|_{x=0}=0, \quad \theta(1)=1 .
\end{gathered}
$$

Integrating over the grid cell $[0, x+\Delta x / 2]$, as discussed previously within the context of the FVM, we obtain

$$
\begin{array}{r}
\int_{0}^{x+\Delta x / 2}\left(\frac{d}{d x}\left(f(x)(1+B \theta) \frac{d \theta}{d x}\right)\right) d x \\
=\mathscr{M}^{2} \int_{0}^{x+\Delta x / 2} \theta^{n+1} d x, \quad 0<x<1,
\end{array}
$$

which is equivalent to

$$
\begin{gathered}
\left(f\left(x+\frac{\Delta x}{2}\right)\left(1+B \theta\left(x+\frac{\Delta x}{2}\right)\right)\right) \frac{d \theta(x+\Delta x / 2)}{d x} \\
=\mathscr{M}^{2} \int_{0}^{x+\Delta x / 2} \theta^{n+1} d x, \quad 0<x<1 .
\end{gathered}
$$


Similarly over $[0, x-\Delta x / 2]$, we find that

$$
\begin{gathered}
\left(f\left(x-\frac{\Delta x}{2}\right)\left(1+B \theta\left(x-\frac{\Delta x}{2}\right)\right)\right) \frac{d \theta(x-\Delta x / 2)}{d x} \\
=\mathscr{M}^{2} \int_{0}^{x-\Delta x / 2} \theta^{n+1} d x, \quad 0<x<1 .
\end{gathered}
$$

By cell averaging over $[0, x+\Delta x / 2]$ and $[0, x-\Delta x / 2]$ and by using the first-order Taylor approximation around $x$ equations (18) and (19), after simple algebraic transformations, give the cell-averaged quantity

$$
\begin{aligned}
& \int_{x-\Delta x / 2}^{x+\Delta x / 2} \theta^{n+1} d x \\
& =\Delta x\left(\theta^{n+1}(x)\right. \\
& \left.\quad+\frac{(n+1) \mathscr{M}^{2} x \Delta x \theta^{2 n+1}(x)}{2 f(x)(1+B \theta(x))-(n+1) \mathscr{M}^{2} x^{2} \theta^{n}(x)}\right) .
\end{aligned}
$$

Equation (20) constitutes our numerical balance law.

Integrating (8) over $[x-\Delta x / 2, x+\Delta x / 2]$ and incorporating expression (20) into the resulting expression we obtain

$$
\begin{aligned}
\frac{\partial \theta}{\partial \tau}= & \frac{1}{(\Delta x)^{2}} \\
& \times\left\{f\left(x+\frac{\Delta x}{2}\right)\left(1+B \theta\left(x+\frac{\Delta x}{2}\right)\right) \frac{\partial \theta(x+\Delta x / 2)}{\partial x}\right. \\
& \left.-f\left(x-\frac{\Delta x}{2}\right)\left(1+B \theta\left(x-\frac{\Delta x}{2}\right)\right) \frac{\partial \theta(x-\Delta x / 2)}{\partial x}\right\} \\
- & \mathscr{M}^{2}\left(\theta^{n+1}(x) \quad(n+1) M^{2} x \Delta x \theta^{2 n+1}(x)\right. \\
& \left.+\frac{(n+1) \mathscr{M}^{2} x^{2} \theta^{n}(x)}{2 f(x)(1+B \theta(x))-(n+1)}\right)
\end{aligned}
$$

where we can see that the order of our equation has been reduced as expected. We now substitute finite difference approximations to our derivatives into (21). We consider $\left[x_{i}-\right.$ $\Delta x / 2, x_{i}+\Delta x / 2$ ] for a particular time $t_{j}$ which provides us with the following approximations:

$$
\begin{gathered}
\left.\frac{\partial \theta\left(x_{i}+\Delta x / 2\right)}{\partial x}\right|_{j}=\frac{\theta^{j}\left(x_{i}+\Delta x\right)-\theta^{j}\left(x_{i}\right)}{\Delta x}=\frac{\theta_{i+1}^{j}-\theta_{i}^{j}}{\Delta x}, \\
\left.\frac{\partial \theta\left(x_{i}+\Delta x / 2\right)}{\partial x}\right|_{j}=\frac{\theta^{j}\left(x_{i}\right)-\theta^{j}\left(x_{i}-\Delta x\right)}{\Delta x}=\frac{\theta_{i}^{j}-\theta_{i-1}^{j}}{\Delta x}, \\
\left.\frac{\partial \theta}{\partial \tau}\right|_{i}=\frac{\theta_{i}^{j+1}-\theta_{i}^{j}}{\Delta t} .
\end{gathered}
$$

Hence, our well-balanced numerical scheme is given by the following recurrence relation:

$$
\begin{aligned}
& \theta_{i}^{j+1} \\
& \begin{array}{c}
=\theta_{i}^{j}+\frac{\Delta t}{(\Delta x)^{2}}\left[f_{i+1 / 2}\left(1+B \theta_{i+1 / 2}^{j}\right)\left(\theta_{i+1}^{j}-\theta_{i}^{j}\right)\right. \\
\left.-f_{i-1 / 2}\left(1+B \theta_{i-1 / 2}^{j}\right)\left(\theta_{i}^{j}-\theta_{i-1}^{j}\right)\right] \\
-\Delta t \mathscr{M}^{2}\left(\left(\theta_{i}^{j}\right)^{n+1}+\frac{(n+1) \mathscr{M}^{2} x_{i} \Delta x\left(\theta_{i}^{j}\right)^{2 n+1}}{2 f_{i}\left(1+B \theta_{i}^{j}\right)-(n+1) \mathscr{M}^{2} x_{i}^{2}\left(\theta_{i}^{j}\right)^{n}}\right),
\end{array}
\end{aligned}
$$

where a linear interpolation is used to determine $f_{i+1 / 2}, f_{i-1 / 2}$, $\theta_{i+1 / 2}$, and $\theta_{i-1 / 2}$.

3.2.1. No Heat Flux Flow at the Fin Tip. In order to implement our well-balanced numerical scheme, we need to first incorporate the relevant boundary conditions. According to the work by Kraus et al. [11], some fins' shapes require special interpretation-a clear example thereof is the triangular fin profile. Longitudinal fins of triangular profile have been classified among singular fins that cannot be characterized by any linear transformations. As such it is important to remember that the fin profile tapers to zero thickness at the tip, and hence, there will be zero flux at this point. This means that

$$
\left.\left(f(x)(1+B \theta) \frac{\partial \theta}{\partial x}\right)\right|_{x=0}=0 .
$$

We now implement a time forward discretisation at the origin and employ the zero-flux condition given by (24) to obtain

$$
\theta_{0}^{j+1}=\theta_{0}^{j}-\Delta \tau \mathscr{M}^{2}\left(\theta_{0}^{j}\right)^{n+1}
$$

As one can see the physical reality of zero thickness at the tip complicates the solution of the problem. If one were to only employ the zero-flux condition, given the initial condition of zero temperature, one would always have a zero temperature at the origin as per (25). This does not make physical sense, however, given that after a considerable time the temperature would be expected to increase at the tip of the fin. At this stage we turn to the well-balancing principle as a means of overcoming this problem.

We employ the well-balancing principle at the origin as a means of incorporating the expression of $\theta_{0}^{j}$ into (25). As such, we consider the steady-sate equation as follows:

$$
\begin{gathered}
\int_{x_{0}}^{x_{0}+\Delta x / 2} \frac{d}{d x}\left(f(x)(1+B \theta) \frac{d \theta}{d x}\right) d x=\frac{\Delta x}{2} \mathscr{M}^{2} \theta^{n+1}\left(x_{0}\right), \\
f\left(x_{0}+\frac{\Delta x}{2}\right)\left(1+B \theta\left(x_{0}+\frac{\Delta x}{2}\right)\right) \frac{d}{d x} \theta\left(x_{0}+\frac{\Delta x}{2}\right) \\
=\frac{\Delta x}{2} \mathscr{M}^{2} \theta^{n+1}\left(x_{0}\right) .
\end{gathered}
$$




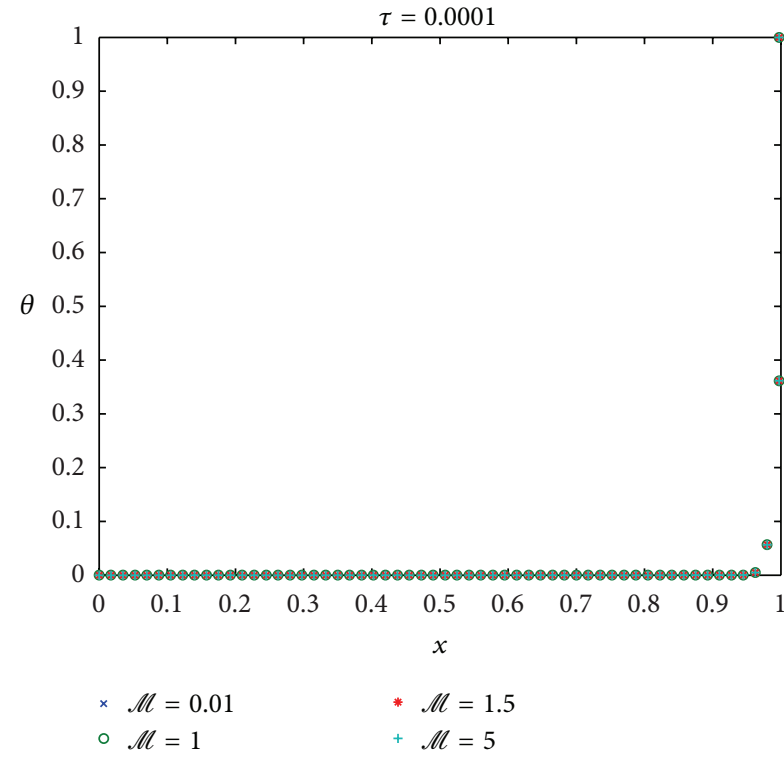

(a)

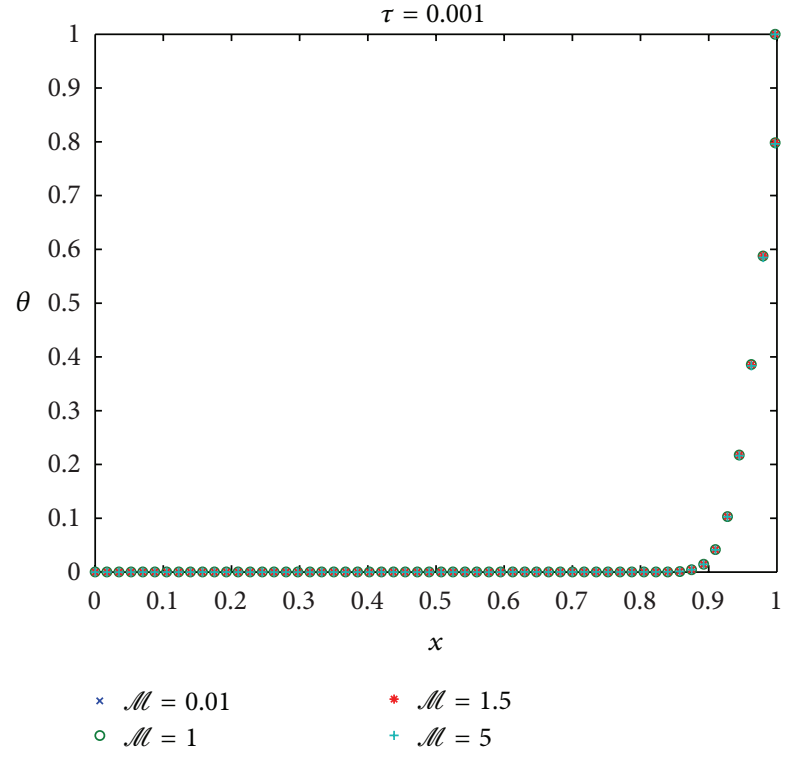

(b)

Figure 2: A triangular fin profile with $B=1, n=1, \mathscr{M}=0.01, \mathscr{M}=1, \mathscr{M}=1.5$, and $\mathscr{M}=5$ for $\tau=0.0001$ (a) and $\tau=0.001$ (b).

Through the use of a central difference approximation, we then obtain

$$
\theta_{0}^{j}=\theta_{1}^{j}-\frac{\Delta x^{2}}{2 f\left(x_{1 / 2}\right)\left(1+B \theta_{1 / 2}^{j}\right)} \mathscr{M}^{2}\left(\theta_{0}^{j}\right)^{n+1}
$$

The coupled equations (25) and (27) provide a numerical well-balanced discretisation for a triangular fin profile at the origin.

3.2.2. Heat Flux Flow at the Fin Tip. For regular fin profiles, the flux at the origin is a finite nonzero value in which case there would be heat flow from the tip. It is difficult to impose such a boundary condition because the nonzero value is not known. Instead we impose an adiabatic boundary condition which allows for this nonzero-flux value at the tip to come out as part of the numerical solution obtained. Thus, at the origin we employ the condition

$$
\left.\frac{\partial \theta}{\partial x}\right|_{x=0}=0
$$

which upon substitution into the equation under consideration gives

$$
\frac{\partial \theta}{\partial \tau}-f(x)(1+B \theta) \frac{\partial^{2} \theta}{\partial x^{2}}+\left.\mathscr{M}^{2}(\theta)^{n+1}\right|_{x=0}=0
$$

Implementing the forward difference approximation for time and the central difference approximation for space, we find that

$$
\theta_{0}^{j+1}=\theta_{0}^{j}+\frac{2 \Delta \tau f\left(x_{0}\right)\left(1+B \theta_{0}^{j}\right)}{\Delta x^{2}}\left(\theta_{1}^{j}-\theta_{0}^{j}\right)-\Delta \tau \mathscr{M}^{2}\left(\theta_{0}^{j}\right)^{n+1},
$$

and using similar finite difference approximations on the steady-state equation of regular fins, we obtain

$$
\theta_{0}^{j}=\theta_{1}^{j}-\frac{\Delta x^{2}}{2 f\left(x_{0}\right)\left(1+B \theta_{0}^{j}\right)} \mathscr{M}^{2}\left(\theta_{0}^{j}\right)^{n+1} .
$$

Equations (30) and (31) summarize the discretisation at the origin for regular fin profiles.

\section{Results and Discussion}

4.1. Triangular Fin Profiles. As stated earlier, previous researches have proposed that one approximates the shape of triangular fins by considering the trapezoidal profile as a means of facilitating linear transformations. Aside from proposed simplifications, work has also been done while maintaining the profile in its original triangular form. In [12] for instance, numerical solutions were obtained for the heat transfer in a triangular fin which did not maintain the adiabatic condition-this was thought to be due to thermal instability within the fin as discussed by Yeh and Liaw [21].

The importance of the work conducted here is that the numerical scheme developed did not rely on any simplifying assumptions as proposed by Kraus et al. [11]. Furthermore, the results obtained in [12] are shown to be due to an inaccurate methodology, specifically related to the boundary conditions for profiles which lead to singularities. In applying the zeroflux condition in a novel manner, we were able to obtain a recursive scheme able to capture the true behaviour of the model under consideration.

We obtained numerical solutions via our well-balanced scheme to (8) for a triangular fin profile with $B=1, n=1$, $\mathscr{M}=0.01,1,1.5$, and $\mathscr{M}=5$ at different values of $\tau$. Figures 2 and 3 indicate that the temperature decreases from the base to 


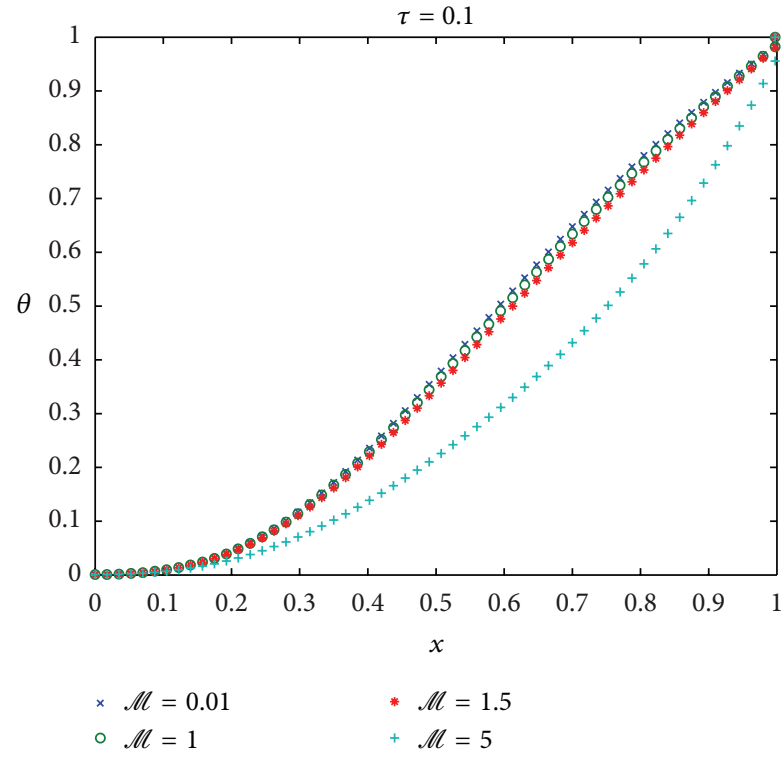

(a)

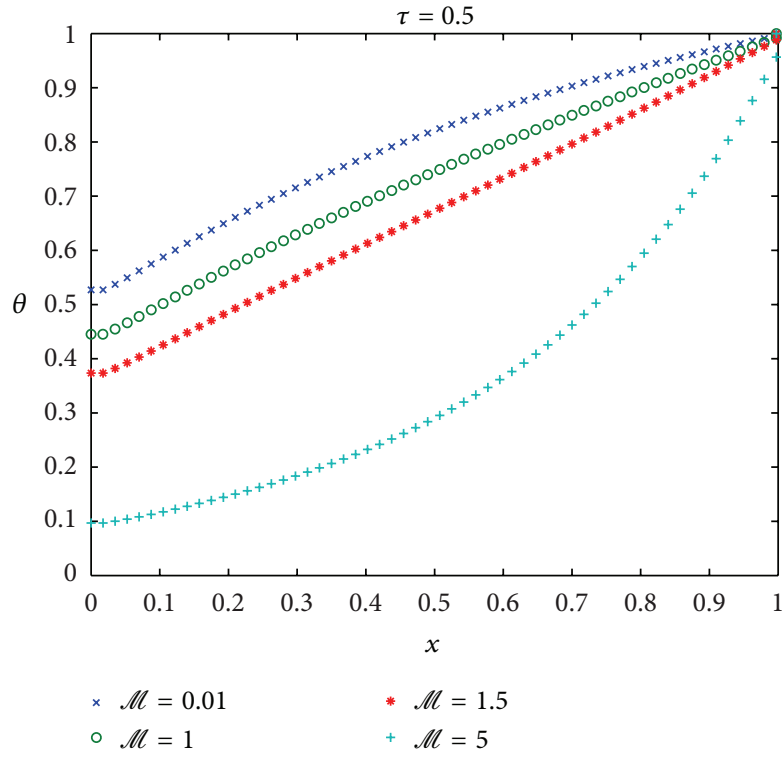

(b)

Figure 3: A triangular fin profile with $B=1, n=1, \mathscr{M}=0.01, \mathscr{M}=1, \mathscr{M}=1.5$, and $\mathscr{M}=5$ for $\tau=0.1$ (a) and $\tau=0.5$ (b).

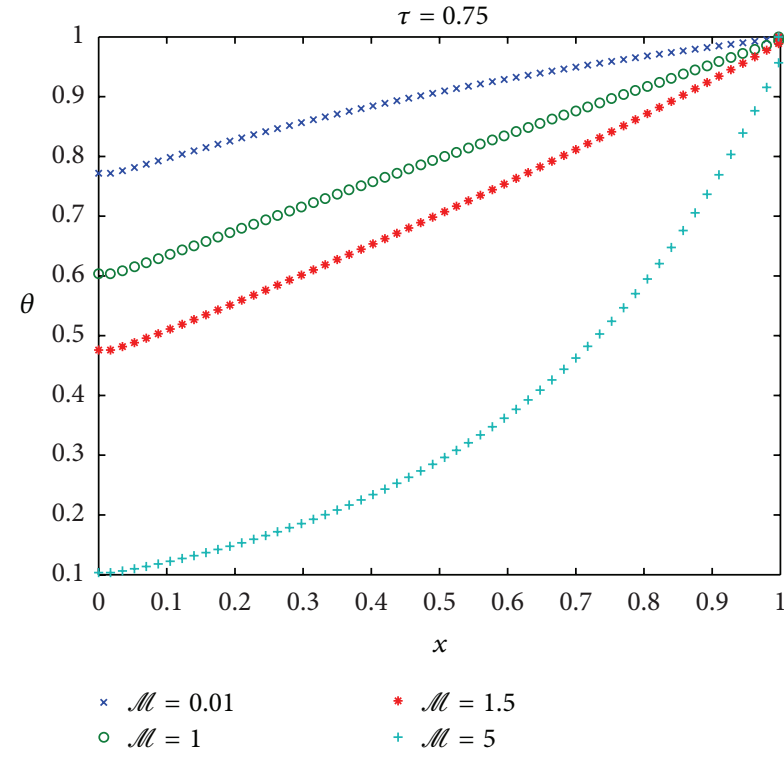

(a)

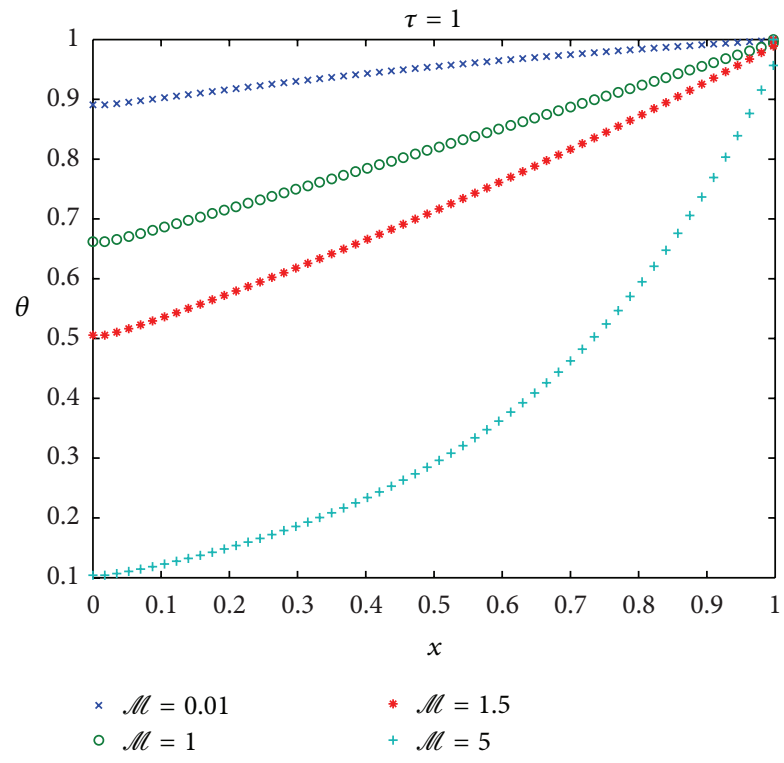

(b)

Figure 4: A triangular fin profile with $B=1, n=1, \mathscr{M}=0.01, \mathscr{M}=1, \mathscr{M}=1.5$, and $\mathscr{M}=5$ for $\tau=0.75$ (a) and $\tau=1$ (b).

tip of the fin and that the temperature at the tip increases with time. These results make physical sense and also match the behaviour of the temperature distribution found for other fin profiles. Interestingly, for small values of time $\tau$, the response temperature is virtually independent of the value of $\mathscr{M}$, and this is why a single curve is shown for $\tau=0.0001, \tau=0.001$, and $\tau=0.01$. This has been explained by Suryanarayana [16] where it is said that at small values of $\tau$, the bulk of the thermal energy entering at the base remains stored in the fin with only a small fraction available for dissipation through surface convection. Thus, the heat transfer coefficient has very little direct impact on the temperature profile-rather its impact may be related to the length of the fin which in turn influences the temperature profile [12].

In turn, as $\tau$ increases, it is seen that the role of convection and hence $\mathscr{M}$ become progressively significant as shown in Figures 4 and 5 . Another point is that the steady state is reached quicker for longer fins or fins with higher values of $\mathscr{M}$ as shown in Figure 3 for $\tau=0.5$. This same Figure 3 shows clearly that at $\tau=0.5$ only a stationary state has been reached for the fin profile where $\mathscr{M}=5$, which is the largest value chosen. This is a consequence of the fact that the 


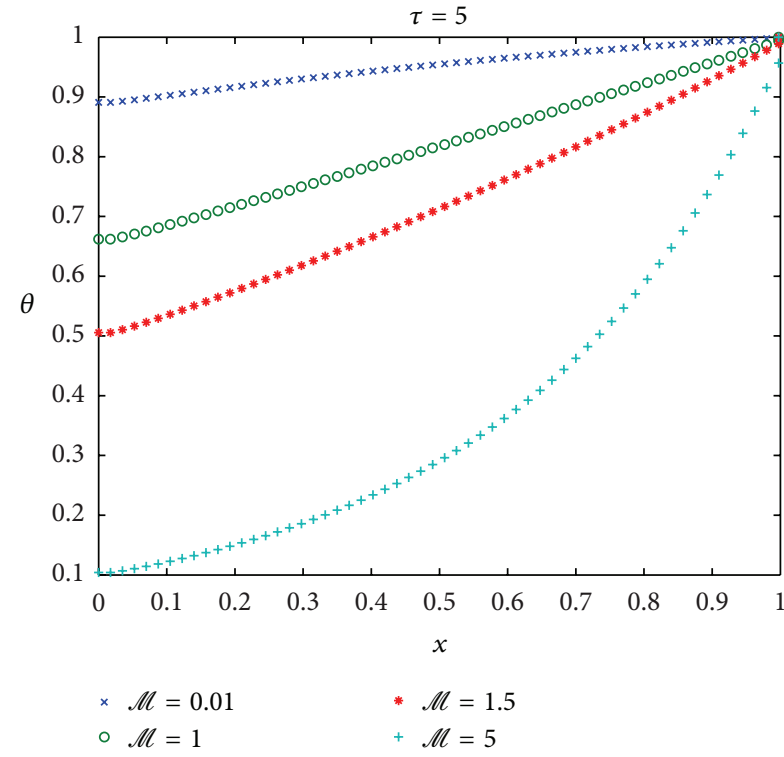

(a)

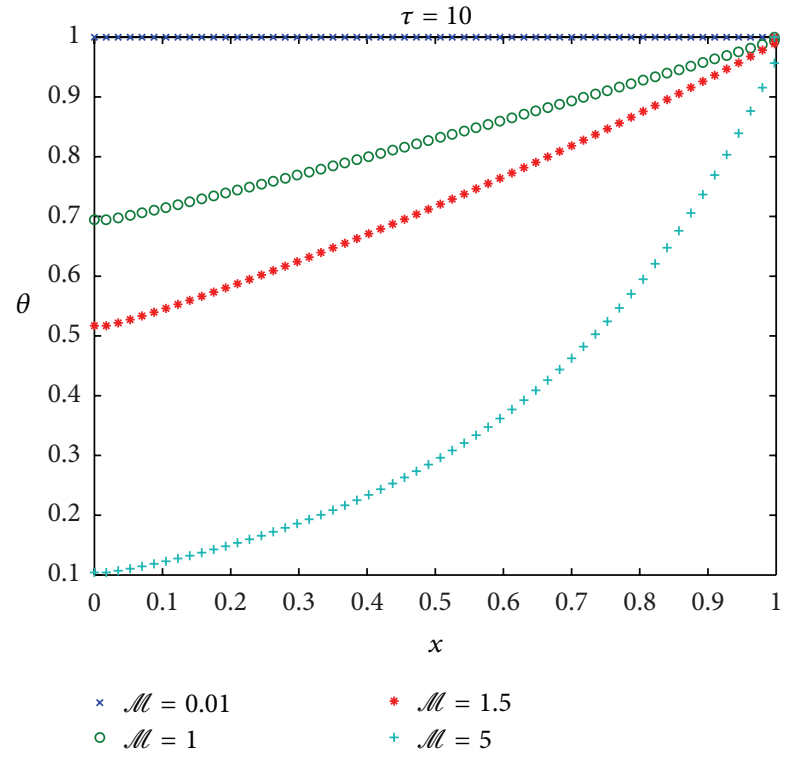

(b)

Figure 5: A triangular fin profile with $B=1, n=1, \mathscr{M}=0.01, \mathscr{M}=1, \mathscr{M}=1.5$, and $\mathscr{M}=5$ for $\tau=5$ (a) and $\tau=10$ (b).

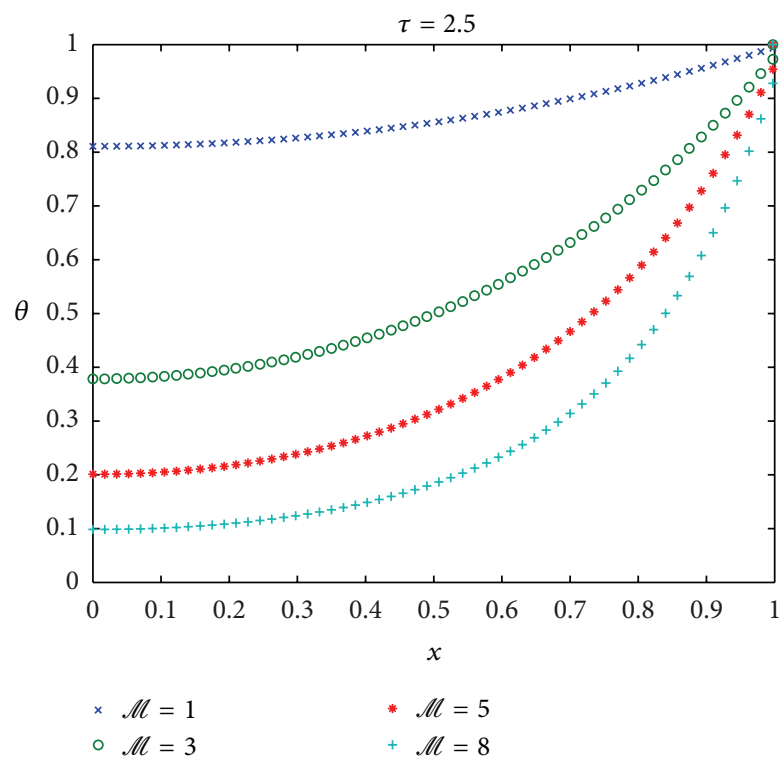

Figure 6: A rectangular fin profile with $B=1, n=1, \mathscr{M}=1, \mathscr{M}=$ 3, $\mathscr{M}=5$, and $\mathscr{M}=8$ for $\tau=2.5$.

dimensionless time $\tau=k_{a} t / \rho c L^{2}$ should decrease with an increase in $\mathscr{M}$.

While the numerical results we have obtained for the heat transfer in triangular fins match those obtained by Suryanarayana [16] for other types of fin profiles in linear cases, we still require further verification of our results. The results provided in [16] in and of itself cannot justify the accuracy of the results obtained via our well-balanced numerical scheme given the fact that no other concrete analysis currently exists and that all previous attempts at obtaining solutions for the

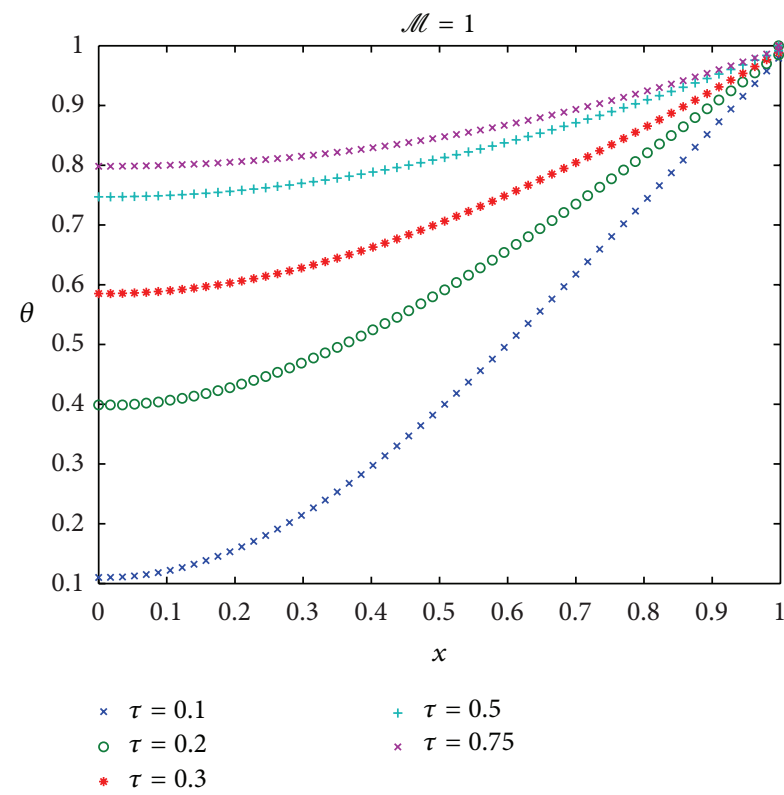

Figure 7: A rectangular fin profile with $B=1, n=1, \mathscr{M}=1$, and varying $\tau$.

triangular case were done with reservations regarding the results obtained [12]. For this reason, our model has been applied to the rectangular case, where we do have confirmed results, as a means of validating the scheme implemented.

4.2. Model Validation via a Consideration of the Rectangular Fin Profile. For a rectangular fin profile, the solution profiles from our well-balanced numerical scheme are depicted by Figures 6,7 , and 8 . It is clearly visible that the temperature 


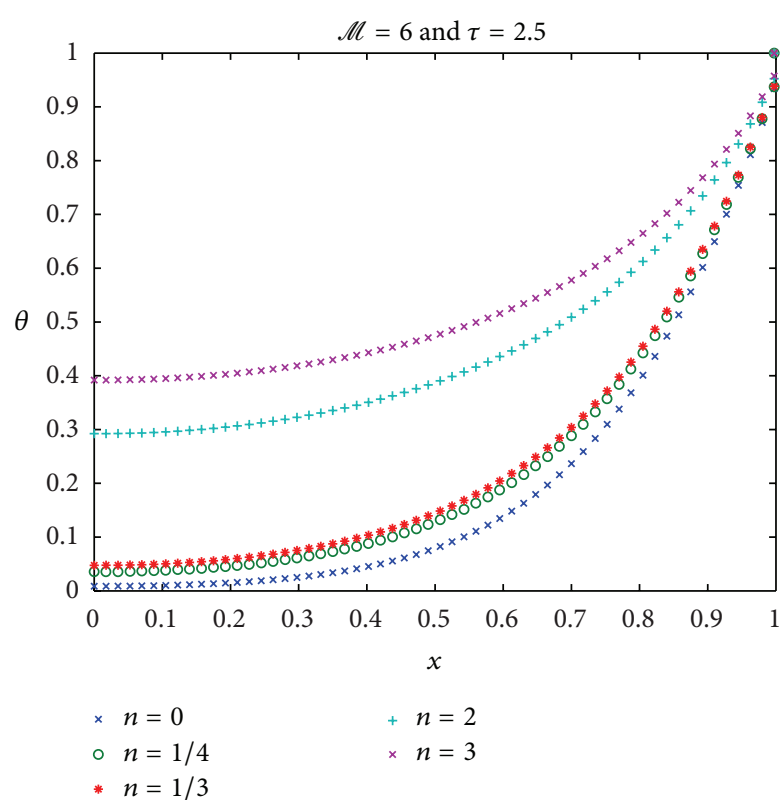

FIGURE 8: A rectangular fin profile with $B=1, \mathscr{M}=6, \tau=2.5$, and varying $n$.

is an increasing function of time and that it decreases from the base to the tip. Figure 6 depicts the effect of the thermogeometric fin parameter on the temperature. We can see that the temperature is a decreasing function of $\mathscr{M}$. In contrast, the temperature distribution is an increasing function of parameter $n$ as shown in Figure 8. What is important to realise is that the results we have obtained for the rectangular case via the well-balanced scheme employed for the triangular case verify the benchmarks results of $[12,16]$ and hence act as a validation of our well-balanced numerical scheme.

\section{Conclusion}

The well-balanced numerical scheme which we have established in this work has been shown to effectively and efficiently obtain results for the rectangular fin profile, matching previous results found in the literature $[12,15,16]$. Our discretisation incorporates the flux condition for the rectangular case as is appropriate; however, for the triangular fin profile, we have incorporated the zero-flux condition into our established well-balanced numerical scheme. It is this latter scheme and the manner in which it is employed which constitute the originality of our work.

Several researchers $[15,20]$ have proposed some exact solutions, but the main problem was that the problem was being simplified through an adjustment of the geometric form of the fin as a means of guaranteeing analytical solutions. Kraus et al. [11], for example, suggested that one assumes triangular profiles to be trapezoidal so as to guarantee the existence of linear transformations.
In our work however, such simplifications are not needed. The well-balanced numerical scheme which we have developed is able to handle the triangular case without any additional assumptions due to the incorporation of an appropriate boundary condition, namely, the zero-flux condition. This approach can easily be extended to other singular profiles, such as the convex and concave parabolic profiles, and hence, it constitutes a clear path to a generalized numerical scheme for the solution of problems in heat transfer.

\section{Nomenclature}

$A_{p}: \quad$ Profile area, $\mathrm{m}^{2}$

B: $\quad$ Thermal conductivity parameter

c: $\quad$ Specific heat capacity, $\mathrm{J} / \mathrm{KgK}$

$c_{v}: \quad$ Volumetric heat capacity $2 c /\left(\delta_{b} A_{p}\right)$

$F(X)$ : Fin profile, $\mathrm{m}^{2}$

$f(x)$ : Dimensionless fin profile

$H$ : $\quad$ Heat transfer coefficient, $\mathrm{W} / \mathrm{m}^{2} \mathrm{~K}$

$h$ : Dimensionless heat transfer coefficient

$h_{b}$ : Heat transfer at the base, $\mathrm{W} / \mathrm{m}^{2} \mathrm{~K}$

K: Thermal conductivity, $\mathrm{W} / \mathrm{mK}$

$k$ : Dimensionless thermal conductivity

$k_{a}$ : Thermal conductivity of the fin at the ambient temperature, $\mathrm{W} / \mathrm{mK}$

$L: \quad$ Length of the fin, $m$

n: Exponent

$P: \quad$ Fin perimeter, $\mathrm{m}$

Q: $\quad$ Heat flux, $\mathrm{W} / \mathrm{m}^{2}$

$T: \quad$ Temperature distribution, $\mathrm{K}$

$T_{b}$ : $\quad$ Fin base temperature, $\mathrm{K}$

$T_{a}$ : $\quad$ Surrounding temperature, $\mathrm{K}$

$t: \quad$ Time, $S$

$X: \quad$ Spatial variable, $\mathrm{m}$

$x$ : $\quad$ Dimensionless spatial variable.

\section{Greek Symbols}

$\beta$ : Thermal conductivity gradient

$\delta$ : Fin thickness, $\mathrm{m}$

$\delta_{b}$ : Fin thickness at the base, $\mathrm{m}$

$\eta:$ Fin efficiency

$\theta$ : Dimensionless temperature

$\mathscr{M}$ : Thermogeometric fin parameter

$\tau$ : Dimensionless time.

\section{Acknowledgments}

C. Harley acknowledges support from the National Research Foundation, South Africa, under Grant no. 79184. Furthermore, this publication was made possible (in part) by a grant from Carnegie Corporation of New York. The statements made and views expressed are, however, solely the responsibility of the authors. 


\section{References}

[1] V. D. Rao, S. V. Naidu, B. G. Rao, and K. V. Sharma, "Heat transfer from a horizontal fin array by natural convection and radiation-a conjugate analysis," International Journal of Heat and Mass Transfer, vol. 49, no. 19-20, pp. 3379-3391, 2006.

[2] E. M. Sparrow and S. B. Vemuri, "Natural convection-radiation heat transfer from highly populated pin-fin arrays," ASME Journal of Heat Transfer, vol. 107, no. 1, pp. 190-197, 1985.

[3] A. Güvenç and H. Yüncü, "An experimental investigation on performance of fins on a horizontal base in free convection heat transfer," Heat and Mass Transfer, vol. 37, no. 4-5, pp. 409-416, 2001.

[4] A. I. Zografos and J. Edward Sunderland, "Natural convection from pin fin arrays," Experimental Thermal and Fluid Science, vol. 3, no. 4, pp. 440-449, 1990.

[5] E. M. Sparrow and S. B. Vemuri, "Orientation effects on natural convection/radiation heat transfer from pin-fin arrays," International Journal of Heat and Mass Transfer, vol. 29, no. 3, pp. 359-368, 1986.

[6] G. Guglielmini, E. Nannei, and G. Tanda, "Natural convection and radiation heat transfer from staggered vertical fins," International Journal of Heat and Mass Transfer, vol. 30, no. 9, pp. 1941-1948, 1987.

[7] H. Yüncü and G. Anbar, "An experimental investigation on performance of rectangular fins on a horizontal base in free convection heat transfer," Heat and Mass Transfer, vol. 33, no. 5-6, pp. 507-514, 1998.

[8] V. Rammohan Rao and S. P. Venkateshan, "Experimental study of free convection and radiation in horizontal fin arrays," International Journal of Heat and Mass Transfer, vol. 39, no. 4, pp. 779-789, 1996.

[9] W. Wang, C. W. Shu, H. C. Yee, and B. Sjogreen, "On wellbalanced schemes fornon-equilibrium flow with stiff source terms," Center for Turbulence Research, Annual Research Briefs, 2008.

[10] R. J. LeVeque, "Balancing source terms and flux gradients in high-resolution Godunov methods: the quasi-steady wavepropagation algorithm," Journal of Computational Physics, vol. 146, no. 1, pp. 346-365, 1998.

[11] A. D. Kraus, A. Aziz, and J. Welty, Extended Surface Heat Transfer, A Wiley-Interscience Publication, New York, NY, USA, 2001.

[12] R. J. Moitsheki and C. Harley, "Transient heat transfer in longitudinal finsof various profiles with temperature-dependent thermal conductivity and heattransfer coefficient," Pramana Journal of Physics, vol. 77, no. 3, pp. 519-532, 2011.

[13] D. Amadori, L. Gosse, and G. Guerra, "Global BV entropy solutions and uniqueness for hyperbolic systems of balance laws," Archive for Rational Mechanics and Analysis, vol. 162, no. 4, pp. 327-366, 2002.

[14] L. Gosse, "Transient radiative transfer in the grey case: wellbalanced and asymptotic-preserving schemes built on Case's elementary solutions," Journal of Quantitative Spectroscopy and Radiative Transfer, vol. 112, no. 12, pp. 1995-2012, 2011.

[15] M. Turkyilmazoglu, "Exact solutions to heat transfer in straight fins of varyingexponential shape having temperature dependent properties," International Journal of Thermal Sciences, vol. 55, pp. 69-75, 2012.

[16] N. V. Suryanarayana, "Transient response of straight fins," ASME Journal of Heat Transfer, vol. 97, no. 3, pp. 417-423, 1975.
[17] F. Khani and A. Aziz, "Thermal analysis of a longitudinal trapezoidal fin with temperature-dependent thermal conductivity and heat transfer coefficient," Communications in Nonlinear Science and Numerical Simulation, vol. 15, no. 3, pp. 590-601, 2010.

[18] F. Khani, M. A. Raji, and H. H. Nejad, "Analytical solutions and efficiency of the nonlinear fin problem with temperaturedependent thermal conductivity and heat transfer coefficient," Communications in Nonlinear Science and Numerical Simulation, vol. 14, no. 8, pp. 3327-3338, 2009.

[19] F. Khani, M. Ahmadzadeh Raji, and S. Hamedi-Nezhad, "A series solution of the fin problem with a temperature-dependent thermal conductivity," Communications in Nonlinear Science and Numerical Simulation, vol. 14, no. 7, pp. 3007-3017, 2009.

[20] R. J. Moitsheki, T. Hayat, and M. Y. Malik, "Some exact solutions of the fin problem with a power law temperature-dependent thermal conductivity," Nonlinear Analysis. Real World Applications, vol. 11, no. 5, pp. 3287-3294, 2010.

[21] R. H. Yeh and S. P. Liaw, "An exact solution for thermal characteristics of fins with power-law heat transfer coefficient," International Communications in Heat and Mass Transfer, vol. 17, no. 3, pp. 317-330, 1990.

[22] N. V. Suryanarayana, "Transient response of straight fins," ASME Journal of Heat Transfer, vol. 97, no. 3, pp. 417-423, 1975.

[23] W. Wang, H. C. Yee, B. Sjogreen, T. Magin, and C.-W. Shu, "Constructionof low-dissipative high-order well-balanced filter schemes for non-equilibrium flows," Center for Turbulence Research, Annual Research Briefs, 2009. 


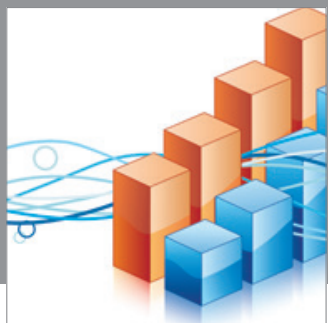

Advances in

Operations Research

mansans

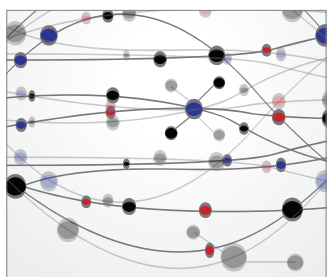

The Scientific World Journal
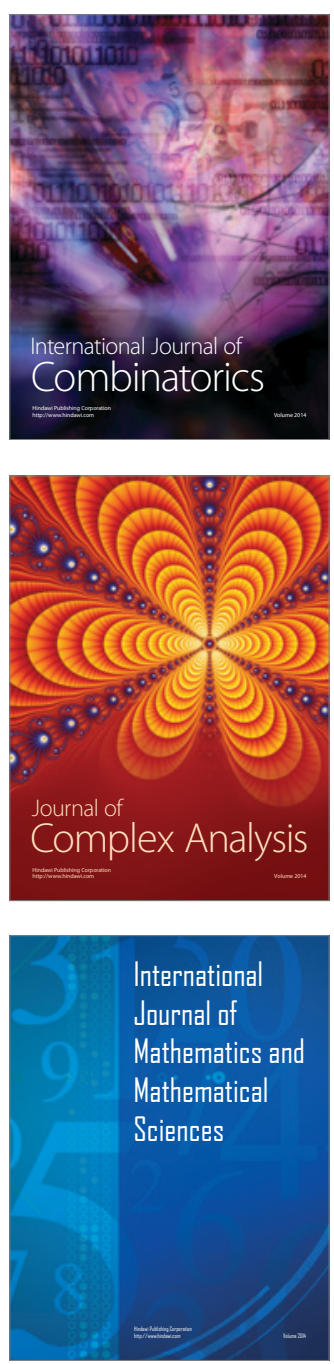
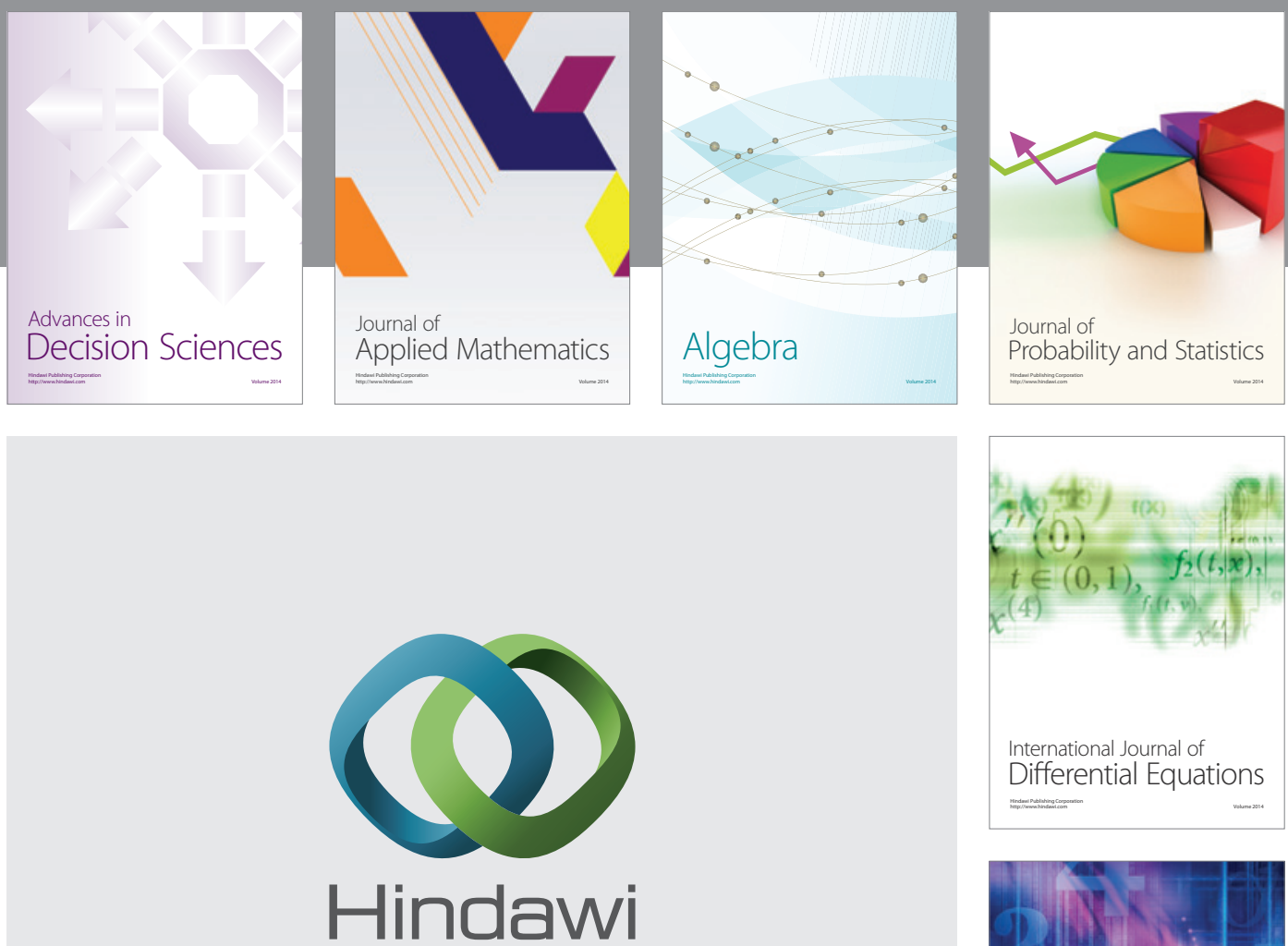

Submit your manuscripts at http://www.hindawi.com
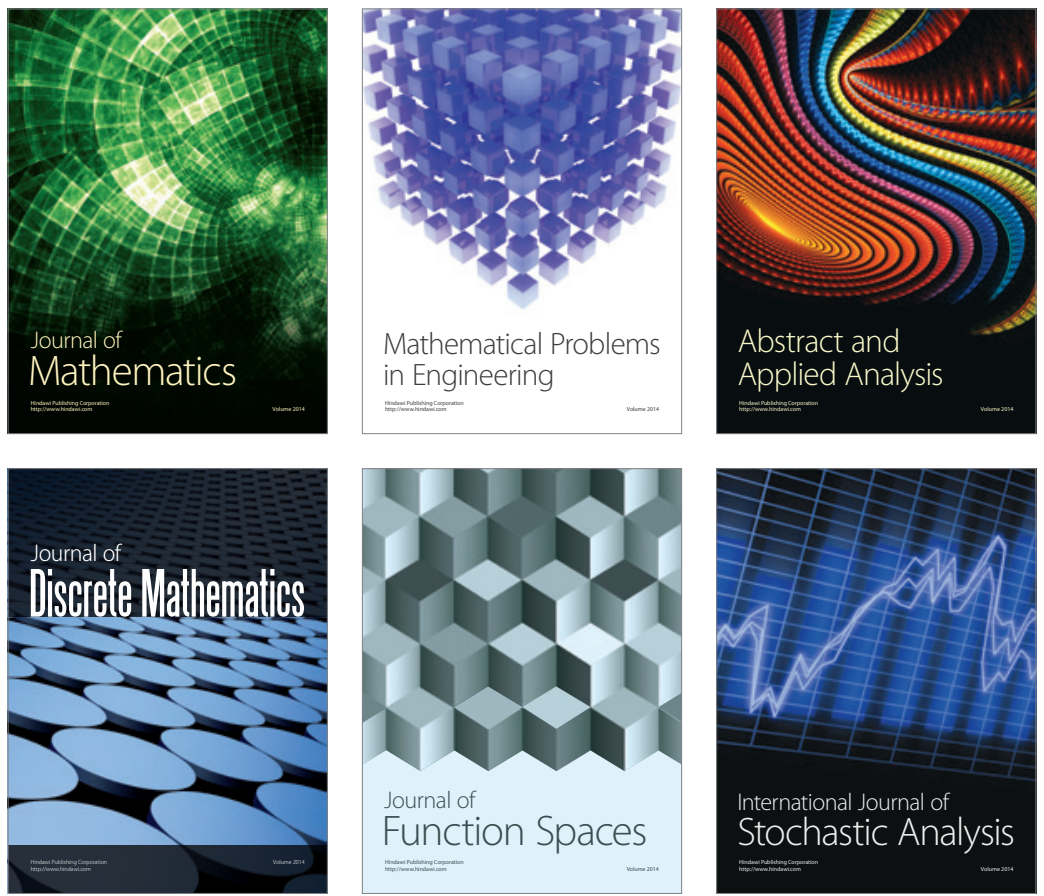

Journal of

Function Spaces

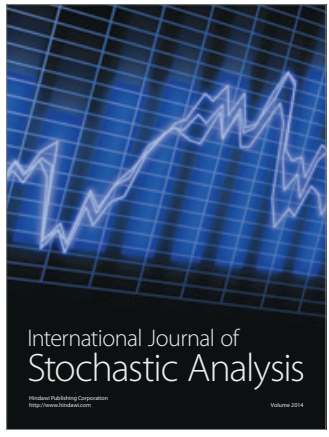

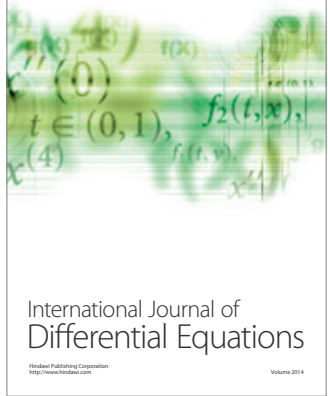
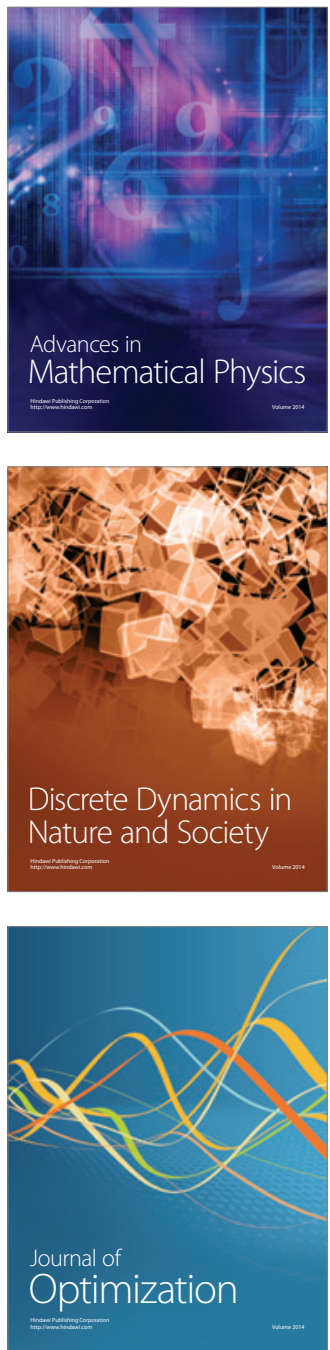